\title{
Cannabis y psicopatología comorbida
}

\author{
Quiroga Gallego, M. \\ Hospital Militar "Capitán Médico Pagés". Melilla, España. \\ Jefe Servicio Psiquiatría. \\ Enviar correspondencia a: \\ Manuel Quiroga Gallego. Servicio de Psiquiatría.Hospital Militar "Capitán Médico Pagés". \\ C/ General Polavieja, s/n.52071 Melilla - España.·Tlf: +34952671145, Ext. 236; E-mail: mquirogag@intersep.org.
}

\section{RESUMEN}

El cannabis, droga ilegal más utilizada por los jóvenes y de las que más precozmente se empieza a consumir, presenta frecuentemente psicopatología comórbida.

Con el cannabis se presentan episodios psicóticos en conciencia lúcida con pocas alteraciones formales del pensamiento, afectividad maniforme, conducta hostil, breve duración y reaparición siempre precedida por su consumo. Sin embargo, sigue en discusión si el cannabis es el detonante de la psicosis o una forma de automedicación de un trastorno psicótico primario.

El uso reiterado desde muy joven de cannabis en sujetos genéticamente vulnerables, causa esquizofrenias cuyo primer episodio se presenta tras un año de consumo de THC, generalmente, antes de los 18 años, con más síntomas positivos y menos negativos que los esquizofrénicos no consumidores, con peor respuesta a los antipsicóticos y más recaídas en los siguientes 15 años. Esto parece ser debido a que en los esquizofrénicos se produce una alteración del sistema cannabinoide endógeno similar a la originada por la intoxicación cannábica en sujetos sanos.

Consumir dosis altas de cannabis durante mucho tiempo conduce a un estado de pasividad e indiferencia caracterizado por disfunción generalizada de las capacidades cognitivas, interpersonales y sociales (llamado síndrome amotivacional) del que empieza a haber evidencias neuropsicológicas, morfológicas y funcionales.

Palabras clave: Cannabis; psicosis; esquizofrenia, patología dual.

\section{INTRODUCCION}

$\mathbf{E}$ título de esta ponencia, lo que primero exige es delimitar el concepto de psicopatología comórbida en el contexto del consumo de drogas.

Por psicopatología comórbida, patología, diagnóstico o trastorno dual se entiende la presencia en el mismo sujeto de una entidad clínica psiquiátrica definida y de una dependencia de una o más drogas.

\section{SUMMARY}

The cannabis, illegal drug more used by the youths and one of those which earlier is begun to consume, it presents frequently comorbid psychopathology.

Consuming cannabis are presented psychotic episodes in lucid conscience with few formal alterations of the thought, maniac feature affectivity, hostile behavior, short duration and reappearance always preceded by its consumption. However, it continues in discussion if the cannabis is the detonative of the psychosis or a form of self-medication of a primary psychotic disorder.

The reiterated use of cannabis from very young, above all in subject genetically vulnerable, cause schizophrenias whose first episode is presented after a yr of THC consumption, generally before 18 years, with more positive symptoms and less negative than schizophrenics not users, with worse response to the antipsychotic and more relapses in the following 15 years. This seems be due to the fact that in the schizophrenics is produced an similar alteration of the endogenous cannabinoid system at the originated by the cannabis-related poisoning in healthly subjects.

To consume high cannabis dose during much time leads to a passiveness and nonchalance state characterized by generalized dysfunction of the cognitives capacities and of the interpersonal and social abilities (call amotivational syndrome) of the that are begun to have neuropsychological, morphologic and functional evidences.

Key words: Cannabis; psychosis; schizophrenia, co-morbidity.
La existencia de esta llamada comorbilidad es sobradamente conocida (1) y afecta más especialmente a los ingresos psiquiátricos (2).

En los adolescentes, las alteraciones psiquiátricas parecen ser un factor etiológico y de vulnerabilidad del consumo de drogas (3).

Casi la mitad de los pacientes mentales jóvenes presentan patología dual y en un tercio de ellos el consumo de drogas ha precedido al diagnóstico de trastorno mental, principalmente al de esquizofrenia (4). 
Pese a estas evidencias y a ser la patología dual una de las principales demandas asistenciales de la década de los 90 (5), su diagnóstico es muy difícil. Esta dificultad es mayor entre los pacientes psicóticos tanto por su frágil psiquismo, fácilmente alterable por las drogas, como por el empleo de potentes psicofármacos, muchas veces de interacciones desconocidas con las drogas al uso (6).

Lo que sí parece evidente es que entre los drogodependientes las tasas de trastorno mental son bajas, en tanto que los pacientes psiquiátricos presentan altos porcentajes de asociación con abuso-dependencia de sustancias (7).

Los estudios epidemiológicos presentan resultados contradictorios tanto en la identificación de casos para la misma población en centros diferentes $(8,9)$, como para los diagnósticos psicopatológicos $(10,11)$. Estas diferencias podrían explicarse porque es el observador quien impone los criterios de inclusiónexclusión, porque existe mayor tendencia en las unidades psiquiátricas para diagnosticar comorbilidad que en las de atención al drogodependiente, pese a tener síntomas psiquiátricos comunes (12) y por la influencia del grado en entrenamiento y metodología en la realización del diagnóstico (13)

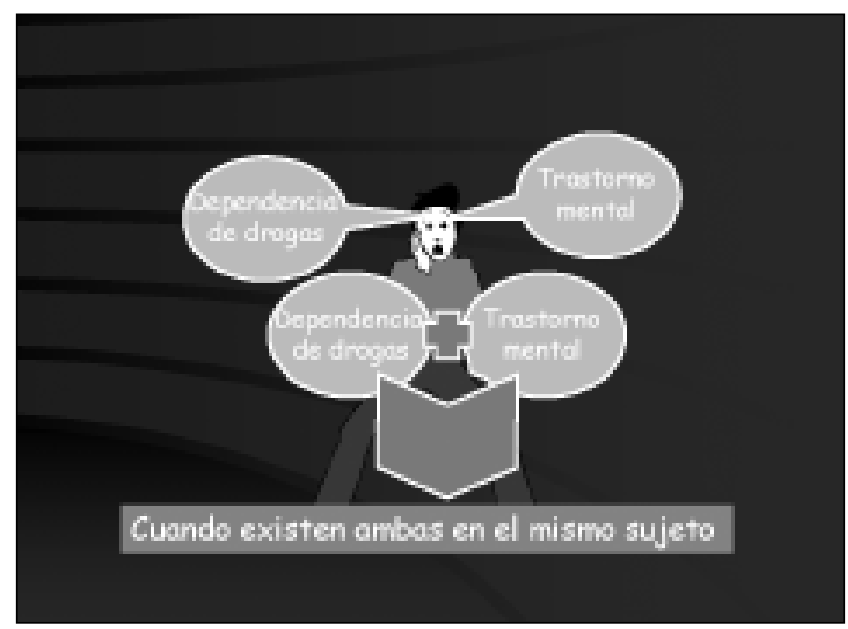

Figura 1.- Concepto de psicopatología comórbida (dual) en drogodependencias

Las tesis etiopatogénicas en patología dual formuladas hacia el final del primer tercio de la década de los 90 eran dos. La similitud entre el déficit neurobioquímico del trastorno mental y la droga (14), la poco reconocida entonces hipótesis de la automedicación. Y la posible existencia de alelos específicos del receptor dopaminérgico D2 en ciertas enfermedades mentales que determinaría una vulnerabilidad para el abuso de drogas (15)

La importancia del diagnóstico dual reside en su trascendencia en cuanto al pronóstico de ambos trastornos, el psiquiátrico y la drogodependencia (16), y a la necesidad de establecer programas de tratamiento adecuados (17-19).

En los estudios más recientes sobre patología dual se aprecia la necesidad de investigar esta comorbilidad separando los sujetos dependientes sólo del alcohol de los policonsumidores de drogas (20-22).

Siguiendo esta línea, llama poderosamente la atención que dos de los últimos estudios publicados, uno realizado en USA y otro en Suiza, pese a su semejanza en objetivos y metodología, sean muy dispares en sus resultados. En tanto que en el estudio americano, pacientes atendidos en un Servicio de Urgencias Psiquiátricas, encuentran asociaciones significativas entre intoxicación aguda y esquizofrenia y trastornos de la personalidad (23), en el suizo, ingresos psiquiátricos en un hospital general, no encuentran afinidad especial entre psicopatología y consumo drogas (24).

En definitiva, estas diferencias podrían explicarse sobre la base de dos abordajes aparentemente distintos. Uno, centrado en la perspectiva de la interacción de las drogas con determinados sistemas neurobiológicos malfuncionantes que resultaría en efectos singulares para la sustancia en cuestión. Y otro, la exposición prolongada a una droga causa una alteración, enfermedad, neurotóxica (25). Ambos puntos no deben contraponerse, sino apreciarlos como complementarios.
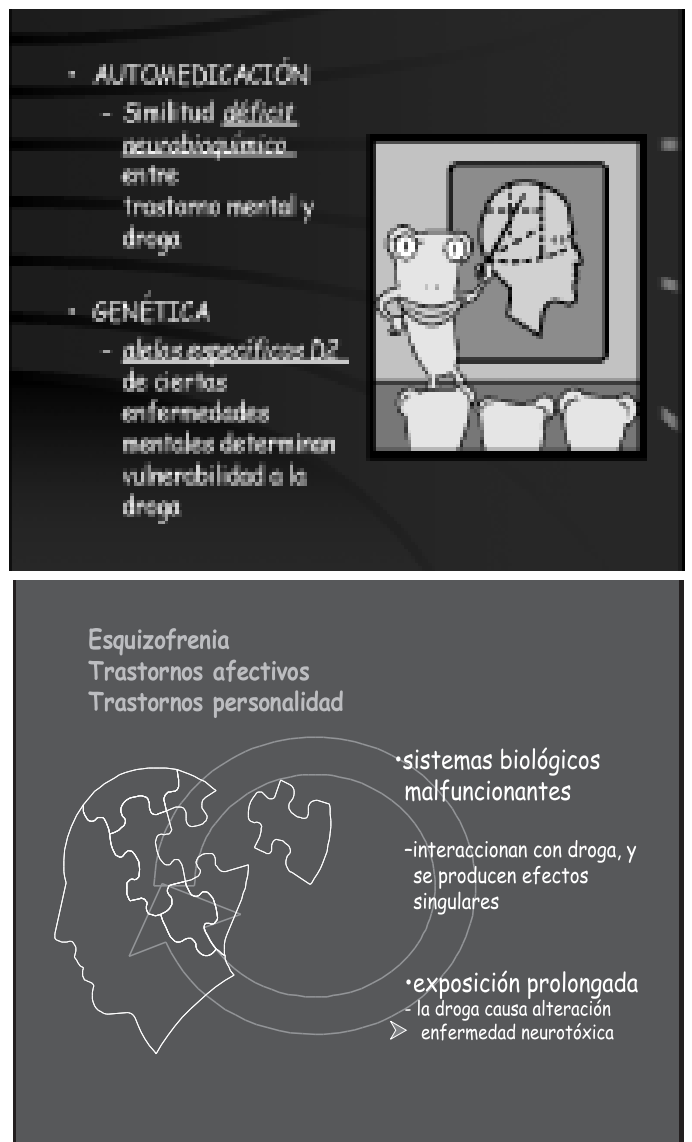

Figura 2.- Etiopatogenia en psicopatología comórbida (dual) 
En el cannabis, el responsable de sus efectos psíquicos es el D9 Tetrahidrocannabinol (THC), cuya concentración en las diferentes preparaciones usadas ("porro", "marihuana", "kiffi", "bhang", "dagga", "ganja", "dawamesc") varía según el tipo de planta y la zona geográfica de cultivo (26).

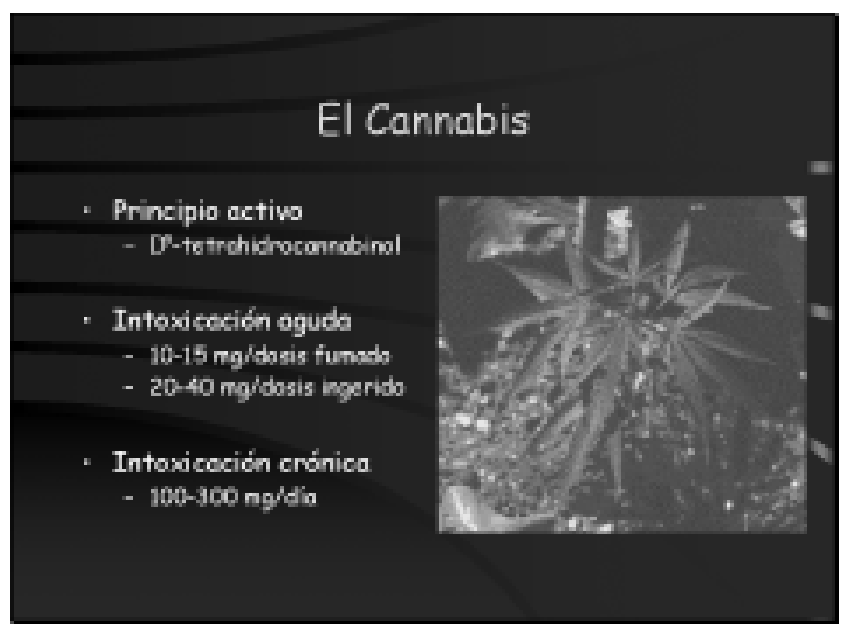

\section{Figura 3.- Intoxicación porTHC. Dosis de} principio activo

La primera publicación científica en occidente sobre los efectos psíquicos del THC, "Du haschich et de l'aliénation mental" (1845), se debe a JacquesJoseph Moreau (1804-1884), médico francés del Hospital Psiquiátrico de La Bicêtre (París), y es fruto de la minuciosa observación del estado que él mismo y sus colaboradores alcanzaban con la ingestión de diferentes dosis de un preparado de cannabis elaborado por el propio Moreau (27). En ella se refieren 8 síntomas que compartían la intoxicación cannábica y las enfermedades mentales (alienación) de la época: euforia, excitación intelectual con disociación de las ideas, distorsión de la percepción del espacio y del tiempo, hipersensibilidad auditiva, ideas fijas y convicciones delirantes, inestabilidad de las emociones, impulsos irresistibles, ilusiones y alucinaciones $(28,29)$ que son dosis dependientes, sin menospreciar la susceptibilidad individual (29).

Otro psiquiatra, Walter Bromberg, americano, basado en su experiencia personal y en multitud de observaciones, describe en 1934, ochenta y nueve años después que Moreau, los efectos psíquicos agudos de la marihuana (cigarrillo de cannabis), cifrando la persistencia de la intoxicación en dos a cuatro horas tras fumarla y en cinco a doce cuando es ingerida (30). Los síntomas descritos por Bromberg, en otro lenguaje, son superponibles a los referidos por Moreau y compartidos por la mayoría de los observadores, con la reserva de que pueden variar ampliamente $y$, generalmente, son menos intensos (31).
Hace 25 años, aproximadamente, los efectos inmediatos directos del THC sobre la salud mental se podían resumir en que, además de los estados de intoxicación aguda (10 a 15 mg de THC fumado; 20 a $40 \mathrm{mg}$ de THC ingerido), su consumo modificaba la presentación de los síntomas en algunas enfermedades mentales (patoplastia), inducía reacciones ansioso-depresivas cuando el consumidor se encontraba en situaciones desfavorables y podía precipitar el inicio de una psicosis latente, en tanto que su uso intenso y prolongado (100 a $300 \mathrm{mg} /$ día) producía en los adultos deterioro mental y físico y en los adolescentes impedía la adecuada maduración de la personalidad (27).

En las más recientes clasificaciones internacionales de los trastornos mentales $(32,33)$ se recogen diversos cuadros psiquiátricos inducidos por cannabis (intoxicación, delirium, alucinatorio), poco específicos pues comparten criterios diagnósticos con otras drogas de naturaleza muy diferentes al THC.

Desde el año 1997, la Organización Mundial de la Salud (OMS) considera que el cannabis incide negativamente en la salud mental porque parece ser capaz de ocasionar un síndrome de dependencia similar al de otras drogas, inducir psicosis propias, causar un síndrome amotivacional, precipitar el inicio y exacerbar el curso de la esquizofrenia (34).

\section{OBJETIVOS}

La presente ponencia se centra en la psicopatología comórbida que se puede presentar junto con la dependencia del cannabis.

Por ello, dejaremos de lado, deliberadamente, las complicaciones físicas y los efectos psíquicos directamente debidos a la acción del THC, sean éstos agudos, síntomas de dependencia o de abstinencia, pues a mi parecer están suficientemente probados (35-40) además de constatarse que en el desarrollo de la dependencia son más importantes los factores genéticos que los ambientales (41-43).

Esto es, nos ocuparemos exclusivamente de las psicosis propias y del síndrome amotivacional por ser entidades en las que el papel del cannabis todavía está en discusión, así como de la incidencia del cannabis sobre el comienzo y curso de la esquizofrenia.

\section{METODOLOGÍA}

Para lograr nuestro objetivo, se ha procedido a la revisión bibliográfica desde 1990 al 2000 buscando en MEDLINE® mediante las palabras claves "dual psychopathology \& cannabis or marijuana". 
El motor de búsqueda empleado ha sido Evaluated Medline ${ }^{\circledR}[($ http://www.biomednet.com/db/medline) (http://www.research.bmn.com/medline)]

\section{RESULTADOS}

La exposición del tema, por razones de claridad, la haremos secuencialmente, aunque esto pueda crear una artificial división en los efectos psicopatológicos comórbidos, ya que, en muchos casos, un trastorno precede, sigue o acompaña al que en ese apartado se describe.

\section{PSICOSIS PROPIAS}

En este apartado consideraremos dos posibilidades que están bajo discusión. Una, episodios psicóticos de breve duración, con lucidez de conciencia, precedidos siempre del consumo de THC. Otra, trastornos psicóticos que persisten más allá de interrumpir el consumo del cannabis y que presentan una clínica y una evolución que permite diferenciarlos de otras psicosis funcionales.

\section{EPISODIOS PSICÓTICOS AGUDOS}

Los episodios psicóticos que aparecen directamente relacionados con el consumo de cannabis, sin disminución del nivel de conciencia, presentan angustia, humor inestable de aspecto hipomaníaco, sentimientos de grandiosidad, aceleración del pensamiento y fuga de ideas, conducta desorganizada y hostilidad, suspicacia, ideación paranoide, alucinaciones no verbales, autolesiones (44-52), siendo algunos de ellos discutidos metodológicamente al no basarse en el estudio controlado de casos, esto es, comparar la psicopatología y el curso del episodio entre sujetos con orinas positivas a cannabinoides frente a negativas (34).

En estudios realizados mediante control de casos, los episodios psicóticos debidos al cannabis, en comparación con los controles libres de THC en orina, presentan más conductas desorganizadas y violentas con pánico y menos alteraciones formales del pensamiento (45); la evolución es más breve y con manifiestos aspectos hipomaníacos (46), semejando un desorden maniforme con elementos paranoides y disfunción cognitiva (49); las diferencias en síntomas al ingreso desaparecen al cabo de una semana (50); tiene menos alteraciones formales del pensamiento, afectividad reactiva y congruente, predomina el polimorfismo en los síntomas clínicos, con corta duración y a las recaídas les precedía siempre el consumo de cannabis (53).
Tabla 1.- Episodios psicóticos agudos'

\begin{tabular}{|c|c|c|c|}
\hline Año estudio & Metodología' & Autor & Referencia $n^{0}$ \\
\hline 1976 & $\begin{array}{l}25 \text { psicosis por cannabis vs } \\
25 \text { esquizofrénicos paranoides }\end{array}$ & Thacore et al. & 45 \\
\hline 1982 & $\begin{array}{l}20 \text { psicóticos THC positivo en } \\
\text { orina vs } 20 \text { psicóticos libres de } \\
\text { cannabis. }\end{array}$ & $\begin{array}{l}\text { Rottanburg et } \\
\text { al. }\end{array}$ & 46 \\
\hline 1991 & $\begin{array}{l}\text { síntomas ingestión bhang en no } \\
\text { psicóticos vs síntomas ingestión } \\
\text { bhang en psicóticos. }\end{array}$ & Chaudry et al. & 49 \\
\hline 1992 & $\begin{array}{l}\text { psicóticos THC positivos en orina } \\
\text { vs psicóticos sin THC, comparando } \\
\text { síntomas a la semana,mes y seis meses }\end{array}$ & Mathers et al. & 50 \\
\hline 1999 & $\begin{array}{l}20 \text { casos de psicosis por cannabis vs } \\
20 \text { casos 'episodio esquizofrénico agudo' }\end{array}$ & Basu et al. & 53 \\
\hline
\end{tabular}

\section{'Sólo estudios con control de casos}

\section{PSICOSIS PERSISTENTES}

El problema subsiste desde el punto en que se hace necesario identificar que las psicosis que acontecen con orinas positivas al THC tienen un inicio, síntomas, curso y respuesta al tratamiento claramente distinta de las demás psicosis funcionales, incluso cuando en éstas intervenga el cannabis (34,54-57).

Estudios en diferentes zonas geoeconómicas y culturales, como Tailandia (58), Jamaica (59), Inglaterra $(60,61)$, Sudáfrica (62), Nigeria (63), Dinamarca (64), concluyen que los estados psicóticos repetidos en consumidores crónicos de THC se presentan principalmente como esquizofrénicos o maníacos y corresponden a una exacerbación y/o modificación patoplástica de la psicosis primaria, con tendencia a la recidiva si se persiste en el consumo de THC.

Un estudio que compara 23 psicóticos con THC positivo en orina frente a 46 psicóticos libres de droga, concluía que las psicosis que se desarrollan o recurren en el contexto del uso del cannabis no tienen características psicopatológicas, ni forma de inicio, ni están restringidas a grupos étnicos, ni sociodemográficos que las distingan de los controles (65).

Más recientemente, se describen dos casos de psicosis con intensas alteraciones formales del pensamiento y de la percepción, delirio y afectividad restringida que se presentaron en ausencia de antecedentes psicóticos, tras consumir continuamente THC durante 8 y 18 meses, respectivamente (66). 
Tabla 2.- Psicosis persistentes ${ }^{2}$

\begin{tabular}{|c|c|c|c|}
\hline \multicolumn{2}{|c|}{ Año publicación } & Autor & Referencia ${ }^{\circ}$ \\
\hline 1994 & $\begin{array}{l}23 \text { psicosis THC positivos orina vs } \\
46 \text { psicóticas libres THC, no existen } \\
\text { diferencias. }\end{array}$ & McGuire et al. & 65 \\
\hline 1999 & $\begin{array}{l}\text { dos casos de psicosis graves tras } \\
\text { consumir } 8 \text { y } 18 \text { meses THC, sin } \\
\text { antecedentes. }\end{array}$ & Leweke et al. & 66 \\
\hline
\end{tabular}

${ }^{2}$ No existen evidencias concluyentes

\section{PSICOSIS ESQUIZOFRENICAS}

La OMS respecto del consumo persistente de THC en relación con la esquizofrenia (34) admite que:

a) precipita su inicio, sobre todo en quienes empezaron con el cannabis antes de los 18 años,

b) aumenta el número y la gravedad de las recaídas en los siguientes 15 años (67-69),

c) siendo más factible cuando el THC se consume sobre una predisposición, vulnerabilidad, esquizofrénica $(58,70-73)$

d) y, también, por la falta de tratamiento adecuado para ambas patologías, la de su dependencia y la de su esquizofrenia (69).

Actualmente, la predisposición o vulnerabilidad esquizofrénica se considera el resultado de la interacción de la organización genética personal con factores de riesgo ambiental, entre los que se incluye el cannabis $(74,75)$.

El por qué consumir THC contribuye a que en la esquizofrenia el inicio sea precoz y mayor el número de recaídas que en las psicosis esquizofrénicas de los no consumidores, empieza a explicarse sobre la base de que el sistema de receptores cannabinoides cerebrales, y sus activadores endógenos, se encuentran también alterados en esquizofrénicos no consumidores de THC.

Mediante pruebas de visión estereoscópica se ha demostrado que la intoxicación cannábica en voluntarios sanos produce alteraciones del sistema de receptores cannabinoides cerebrales, y sus activadores endógenos, similares a las observadas en pacientes esquizofrénicos no consumidores de THC en $(76,77)$.

Más recientemente, comparando la concentración de cannabinoides endógenos (anandamida y plamitiletanolamida) en líquido cefalorraquídeo de 10 pacientes con esquizofrenia con 11 no esquizofrénicos (controles), se encontraron niveles significativamente mayores en el grupo de esquizofrénicos que en los controles, sin que estos resultados estuvieran condicionados ni por el sexo, ni por la edad ni por la medicación antipsicótica (78).

Tabla 3.- Cannabis y esquizofrenia

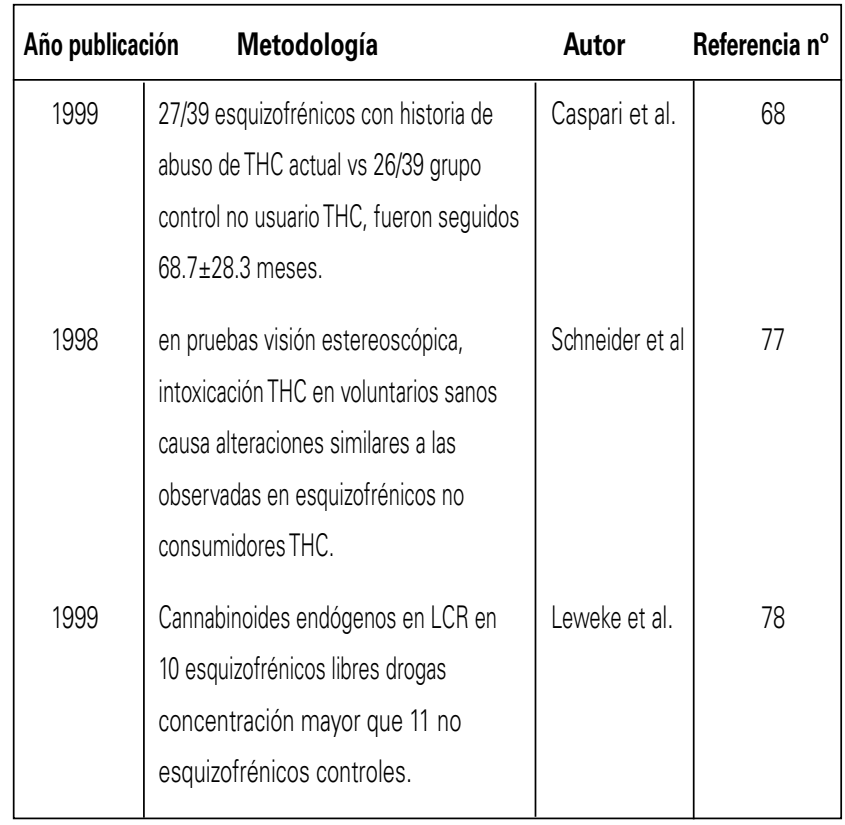

El consumo de THC precipita el inicio a edad temprana de la esquizofrenia y agrava su curso.

La acción del cannabis parece mediada por el sistema cannabinoide endógeno.

\section{SÍNDROME AMOTIVACIONAL}

Por 'síndrome amotivacional' se entiende un estado de pasividad e indiferencia, caracterizado por disfunción generalizada de las capacidades cognitivas, interpersonales y sociales (29), debido al consumo de THC durante años que persistiría una vez interrumpido dicho consumo.

Para la OMS, sigue sin estar suficientemente diferenciado este "síndrome amotivacional" de los efectos directos de la intoxicación crónica por THC (34).

Al comparar consumidores diarios durante años $(6$ años de media) con otros que lo hacían varias veces al mes durante menos tiempo (4.5 años de media), parece ser que los síntomas que en el primer grupo se atribuyen al síndrome amotivacional corresponderían a una depresión (79).

Hay autores que consideran que el síndrome amotivacional no sólo lo causa el consumo prolongado de altas dosis de THC, sino también, las anfetaminas, la cocaína y los solventes volátiles orgánicos (80).

Y es, precisamente, en usuarios crónicos de solventes orgánicos volátiles que se han descrito alteraciones del flujo cerebral, medido con tomografía 
computarizada por emisión única de fotones (SPECT), en el córtex frontal de ambos hemisferios cerebrales correlacionando la intensidad de la disminución del flujo con el incremento de la puntuación en la escala "abolición-apatía" del SANS (Scale for the Assessment of Negative Symptoms) (81). También se ha encontrado esta correlación entre la disfunción frontal identificada mediante examen neuropsicológico y la hipoperfusión del córtex de los lóbulos frontales especialmente en su zona medial (82).

Respecto al cannabis, su consumo repetido reduce el flujo cerebral (SPECT) en ambos lóbulos temporales (83) y altera el trazado del electroencefalograma (EEG) de forma tal que en consumidores diarios de THC entre 15 a 24 años en comparación con no consumidores y consumidores durante menos tiempo, se encontró que, además de la hiperfrontalidad alfa, la actividad theta se alteraba bilateralmente en la región fronto-central, al tiempo que incrementaba su coherencia interhemisférica en zonas centrales y posteriores (84), con marcada reducción de la actividad alfa en aquellos que habían estado consumiendo diariamente durante más años (85).

Continuar investigando correspondencias entre síntomas negativos y variaciones regionales en el flujo sanguíneo cerebral y en la actividad bioeléctrica cerebral, sea, quizás, el camino para dilucidar si realmente existe un síndrome amotivacional inducido por cannabis.

Tabla 4.- Síndrome amotivacional

\begin{tabular}{|c|c|c|c|c|}
\hline \multicolumn{2}{|c|}{ Año publicación } & Metodología & Autor & Referencia $\mathrm{n}^{0}$ \\
\hline 1995 & & $\begin{array}{l}\text { liario } 6 \text { años vs 4,5 años: } \\
\text { nas primer grupo es una } \\
\text { sión. }\end{array}$ & Musty et al. & 79 \\
\hline 1998 & & $\begin{array}{l}\text { educe flujo sanguíneo cerebral } \\
\text { bos lóbulos temporales (SEPCT). }\end{array}$ & Amen et al. & 83 \\
\hline 1999 & & $\begin{array}{l}\text { onsumo crónico altera EEG: } \\
\text { ontalidad alfa, hipersincronía theta } \\
\text { ral, reducción frecuencia alfa. }\end{array}$ & Struve et al. & 85 \\
\hline
\end{tabular}

Comienzan a establecerse las bases morfofuncionales del síndrome

\section{DISCUSIÓN}

La comorbilidad del cannabis parece ser un hecho cada vez más demostrado.

Hay evidencias de que la patología mental entre los 15 y 18 años es un factor de riesgo para la depen- dencia del cannabis, en tanto que ésta entre los 18 y 21 años lo es para la patología mental en la edad adulta (86)

Sigue siendo muy difícil identificar psicosis ocasionadas por cannabis. Parece ser que éstas, para producirse, precisan de la concurrencia del consumo de preparaciones con alto contenido de THC, durante prolongados períodos de tiempo y la existencia de una predisposición (ciertamente inespecífica) en el sujeto (87).

Sin embargo, el consumo de THC sí tiene una clara influencia sobre el inicio y curso de la esquizofrenia (87) En este sentido, el inicio en el abuso de THC y el primer brote esquizofrénico establece tres grupos: a) el consumo de cannabis precede en años al primer episodio esquizofrénico, b) que ambos suceden en el mismo mes, y c) que el inicio del consumo de THC es muy posterior a los primeros síntomas de esquizofrenia (88).

Como posibles factores que condicionarían la comorbilidad esquizofrenia-cannabis, es importante tener en cuenta la asociación entre acusados rasgos de esquizotipia y déficits atencionales, similares a los que se presentan en la fase aguda de la esquizofrenia, observados en los consumidores de THC $(89,90)$.

Sobre su acción en el sistema cannabinoide endógeno, la esquizofrenia y el consumo de cannabis parecen mostrarse independientes. Así, en sujetos esquizofrénicos, independientemente de que hayan consumido o no cannabis, aparece un incremento del receptor cannabinoide endógeno 1 (CNR1) en el área 9 de Brodman, en tanto que en el consumo de cannabis, sin relación con el diagnóstico psiquiátrico, el incremento del CNR1 se localiza en el área del núcleo caudado y putamen (91), lo que mantiene abierta la posibilidad de que el THC cause trastornos psicóticos específicos.

En cuanto al síndrome amotivacional, parece reconocerse que es un estado de deterioro común a muchas sustancias psicotropas, y que morfofuncionalmente (MRI, SPECT, exámenes neuropsicológicos) se caracteriza por cambios en la sustancia blanca, hipoperfusión en la corteza frontal del cerebro y disminución del funcionalismo de lóbulo frontal (92).

\section{CONCLUSIONES}

El cannabis, la droga ilegal más utilizada por la población joven y una de las que más precozmente se empieza a consumir, es frecuentemente encontrada como psicopatología comórbida.

Se presentan episodios psicóticos agudos sin alteración del nivel de conciencia que se distinguen de las psicosis funcionales porque tienen menos alteracio- 
nes formales del pensamiento, la afectividad suele ser reactiva, congruente y tomar un aspecto maniforme, la conducta es a menudo hostil, la duración es breve y su reaparición siempre va precedida del consumo de THC.

Sin embargo, no se ha podido establecer todavía una psicosis persistente por acción exclusiva del cannabis, pues sigue en discusión si el cannabis es detonante, agravante, mero acompañante o forma de automedicación de un trastorno mental primario.

El uso reiterado desde edades muy jóvenes de cannabis en sujetos genéticamente vulnerables, sobre todo si coincide predisposición para depender de la droga y para la esquizofrenia, lleva a psicosis esquizofrénicas cuyo primer episodio se presenta, generalmente, antes de los 18 años tras un año de consumo de THC, con más síntomas positivos y menos negativos que los esquizofrénicos no consumidores, con peor respuesta a los antipsicóticos y más recaídas en los siguientes 15 años.

La razón de esta particular influencia del THC en la esquizofrenia parece ser debida a que en los esquizofrénicos se produce una alteración del sistema cannabinoide endógeno similar a la originada por la intoxicación cannábica en sujetos sanos.

Consumir dosis altas de THC durante mucho tiempo conduce a un estado de pasividad e indiferencia caracterizado por disfunción generalizada de las capacidades cognitivas, interpersonales y sociales (llamado síndrome amotivacional) del que empieza a haber evidencias neuropsicológicas, morfológicas y funcionales.

\section{BIBLIOGRAFÍA}

1 Llaquet Baldellou L; Quiroga Gallego M. Patología Mentaly Toxicofilias. Medicina Militar 1986;Vol.42, n 4

2 Galanter M; Castaneda R; Ferman J. Substance abuse among general psychiatric patients: place of presentation, diagnosis, and treatment. Am J brug Alcohol Abuse 1988;14(2):211-35

3 Bukstein OG; Brent bA; Kaminer Y. Comorbidity of substance abuse and other psychiatric disorders in adolescents. Am J Psychiatry1989 Sep;146(9):1131-41

4 Caton CL; Gralnick A; Bender 5; Simon R. Young chronic patients and substance abuse. Hosp Community Psychiatry 1989 Oct; $40(10): 1037-40$

5 Westermeyer J. Substance use disorders: predictions for the 1990s. Am J brug Alcohol Abuse 1992;18(1): $1-11$

6 Ries RK. Serial, parallel, and integrated models of dualdiagnosis treatment. J Health Care Poor Underserved 1992 Summer; 3(I):173-80
7 Miller NS. Comorbidity of psychiatric and alcohol/drug disorders: interactions and independent status. J Addict bis 1993;12(3):5-16

8 Sanguineti $\sim$ Samuel SE. Reported prevalence of drug abuse comorbidity in a city-wide emergency rooms system. Am J brug Alcohol Abuse 1993;19(4):443-50

9 Lehman AF; Myers CP; Thompson JW; Corty E. Implications of mental and substance use disorders. A comparison of single and dual diagnoSiS patients. J Nerv Ment bis 1993 Jun;181(6):36-70.

10 Sanguineti VR; Samuel SE. Comorbíd substance abuse and recovery from acute psychiatric relapse. Hosp Community Psychiatry 1993 Nov;44(I1):1073-6

11 Westermeyer J; Neider J; Westermeyer M. Substance use and other psychiatric disorders among 100 American Indian patients. Cult Med Psychiatry 1992-93;16(4): 519-29

12 Raskin Vb; Miller NS. The epidemiology of the comorbidity of psychiatric and addictive disorders: a critical review. J Addict bis 1993; 12(3):45-57

13 Kranzler Hk Kadden RM; Burleson JA; Babor TF; Apter A; Rounsaville BJ. Validity of psychiatric diagnoses in patients with substance use disorders: is the interview more important than the interviewer? Compr Psychiatry 1995 Jul-Aug; 36(4): 278-88

14 Extein IL; Gold MS. Hypothesized neurochemical models for psychiatric syndromes in alcohol and drug dependence. J Addict bis 1993;12(3):29-43

15 Becker KP; Ries RK. bifferential diagnosis and psychopharmacology of dual disorders. Psychiatr Clin North Am 1993 Dec;16(4): 703-18

16 Lehman AF; Myers CP; Corty E; Thompson JW. Prevaience and patterns of "dual diagnosis" among psychiatric inpatients. Compr Psychiatry 1994 Mar-Apr; 35(2):106-12

17 Lehman AF; Myers CP; Corty E; Thompson J Severity of substance use disorders among psychiatric inpatients. J Nerv Ment bis 1994 Mar;182(3):164-7

18 Lehman A.F.; Myers C.P.; Bixon L., Johnson J.L. Befining subgroups of dual diagnosis patients for service planificación. Hosp Community Psychiatry 1994 Jun; 45(6): 556-61

19 Ries R; Mullen M; Cox G. Symptom severity and utilization of treatment resources among dually diagnosed inpatients. Hosp Community Psychiatry 1994 Jun; 45(6):562-8

20 Tómasson K.; Vaglum P. A. nationwide representative sample of treatment-seeking alcoholics: a study of psychiatric comorbidity. Acta Psychiatr Scand 1995 Nov;92(5): 378-85

21 Piazza NJ. Dual diagnosis and adolescent psychiatric inpatients. Subst Use Misuse 1996 Jan; 31(2):215-23.

22 Lambert MT; Griffith JM; Hendrickse W. Characteristics of patients with substance abuse diagnoses on a general psychiatry unit in a VA Medical Center. Psychiatr Serv 1996 Oct;47(10):1104-7

23 Breslow RE; Klinger BI; Erickson BJ. Acute intoxication and substance abuse among patients presenting to a 
psychiatric emergency service. Gen Hosp Psychiatry 1996 May; 18(3):183-91

24 Westermeyer J; Eames SE. Clinical epidemiology of comorbid dysthymia and substance disorder. Am J Addict 1997 Winter; 6(1): 48-53

25 Alamo C, López-Muñoz F, Cuenca E. Fundamentos neurobiológicos del trastorno dual: hipótesis etiopatogénicas. En Salvanés A, Álamo C, editores de Avances en patología dual. Aspectos diagnósticos, clínicos, terapéuticos y asistenciales. Servicio de Publicaciones. Universidad de Alcalá, Madrid, 1999;143-62.

26 Beneit Montesinos JV, Portoles Pérez A, Lizasoain Hernández I. Características químicas y farmacocinéticas de los cannabinoides. En Beneit Montesinos JV, López Corral JC, editores de "Drogodependencias. Aspectos farmacológicos y clínicos", Escuela Universitaria de Enfermería, Fisioterapia y Podología, Universidad Complutense de Madrid, 1990:167-81

27 Nahas G. Haschis, cannabis et marijuana. Premièr Edition, Vendôme (France), Presses Universitaires de Fance, 1976:243-95

28 Gold MS. Marihuana. Ediciones en Neurociencias, Primera Edición, Barcelona, 1991:91-101

29 Grinspoon L. Dependencia de las drogas: agentes no narcóticos. En Freedman AM, Kaplan HI, Sadock BJ, editores de "Tratado de psiquiatría", Salvat Editores S.A., Barcelona 1982; II(23.2):1468-73

30 Grinspoon L. Dependencia de las drogas: agentes no narcóticos. En Freedman AM, Kaplan HI, Sadock BJ, editores de "Tratado de psiquiatría", Salvat Editores S.A., Barcelona 1982;II(23.2):1470

31 Grinspoon L, Bakalar JB. Drogodependencia: agentes no narcóticos. En Kaplan HI, Sadock BJ, Cancro R, directores de "Tratado de Psiquiatría", Segunda Edición. Salvat Editores S.A., Barcelona 1989:II(22.2):995-1007

32 Organización Mundial de la Salud. F10-F19 Trastornos mentales y del comportamiento debidos al consumo de sustancias psicotropas. En "Décima revisión de la Clasificación Internacional de Enfermedades. Trastornos mentales y del comportamiento. Descripciones clínicas y pautas para el diagnóstico". Meditor y JJ López Ibor, Madrid 1992:97-112

33 American Psychiatric Association. Trastornos relacionados con Cannabis. En "DSM-V. Manual diagnóstico y estadístico de los trastornos mentales. Cuarta Revisión". Edición española Salvat Editores S.A. Barcelona 1995:227-33

34 Division of mental health and prevention of substance abuse. World Health Organization. Cannabis: a health perspective and research agenda. WHO/MSA/PSA/ 97.4 English Only Distr.: General. Geneva 1997

35 Wiesbeck GA, Schuckit MA, Kalmijn JA, Tipp JE, Bucholz KK, Smith TL. An Evaluation of the History of a Marijuana Withdrawal Syndrome in a Large Population. Addiction 1996;91(10):1469-1478

36 Crowley TJ, Macdonald MJ, Whitmore EA, Mikulich, SK. Cannabis dependence, withdrawal, and reinforcing effects among adolescents with conduct symptoms and substance use disorders. Drug Alcohol Depend 1998; 50(1):27-37

37 Taylor HG. Analysis of the medical use of marijuana and its societal implications. J Am Pharm Assoc (Wash) 1998; 38(2):220-7

38 Kobayashi H, Suzuki T, Kamata R, Saito S, Sato I, Tsuda S, Matsusaka N. Recent progress in the neurotoxicology of natural drugs associated with dependence or addiction, their endogenous agonists and receptors. J Toxicol Sci 1999; 24(1):1-16

39 Haney M, Ward AS, Comer SD, Foltin RW, Fischman $\mathrm{MW}$. Abstinence symptoms following smoked marijuana in humans. Psychopharmacology (Berl) 1999; 141 (4) : 395-404

40 Haney M, Ward AS, Comer SD, Foltin RW, Fischman MW. Abstinence symptoms following oral THC administration to humans. Psychopharmacology (Berl) 1999; 141(4):385-94

41 Organización Mundial de la Salud. Libro de casos de la CIE-10: Las diversas caras de los trastornos mentales: Historias clínicas de casos de trastornos mentales y del comportamiento en personas adultas, comentadas según las descripciones de la ClE-10. Editorial Médica Panamericana S.A., Madrid 1999:62-3

42 van den Bree MB, Johnson EO, Neale MC, Pickens RW. Genetic and environmental influences on drug use and abuse/dependence in male and female twins. Drug Alcohol Depend 1998;52(3):231-41

43 Kendler KS, Prescott CA. Cannabis use, abuse, and dependence in a population-based sample of female twins. Am J Psychiatry 1998; 155(8):1016-22

44 Farini Duggan J, Aust M. Marihuana psychoses. Acta Psiquiatr Psicol Am Lat 1976; 22(1):63-70

45 Thacore VR, Shukla SR. Cannabis psychosis and paranoid schizophrenia. Arch Gen Psychiatry 1976;33(3):383-6

46 Rottanburg D, Robins AH, Ben-Arie O, Teggin A, Elk R. Cannabis-associated psychosis with hypomanic features. Lancet 1982;2(8312):1364-6

47 Thornicroft G. Cannabis and psychosis. Is there epidemiological evidence for an association? Br J Psychiatry 1990; 157:25-33. [published erratum appears in $\mathbf{B r} \mathbf{J}$ Psychiatry 1990;157:460]

48 Crespo MD, Morales MP, Vinas R, Rios B, Ruiz L, Franco B. Psicosis y consumo de cannabis: estudio de las diferencias psicopatológicas y factores de riesgo. Actas Luso Esp Neurol Psiquiatr Cienc Afines 1990; 18(2):120-4

49 Chaudry HR, Moss HB, Bashir A, Suliman T Cannabis psychosis following bhang ingestion. Br J Addict 1991; 86(9):1075-1081

50 Mathers DC, Ghodse AH. Cannabis and psychotic illness. Br J Psychiatry 1992; 161:648-53

51 Quiroga Gallego M. Cannabis et psychopathologie parmis les jeunnes hommes. Types de troubles mentaux avec la cannabis positive dans I'urine. Europ Psychiatry 1996; $11(4): 327 \mathrm{~s}$

52 Sembhi S, Lee JW. Cannabis use in psychotic patients. Aust N Z J Psychiatry 1999; 33(4):529-32 
53 Basu D, Malhotra A, Bhagat A, Varma VK. Cannabis psychosis and acute schizophrenia. a case-control study from India. Eur Addict Res 1999; 5(2):71-3

54 Negrete JC. Effect of cannabis use on health. Acta Psiquiatr Psicol Am Lat 1983; 29(4):267-76

55 Court JM. Cannabis and brain function J Paediatr Child Health 1998; 34(1):1-5

56 Campbell J. Cannabis: the evidence. Nurs Stand 1999; 13(44):45-7

57 Ungerleider JT. Marijuana: still a "signal of misunderstanding". Proc Assoc Am Physicians 1999; 111 (2):173-81

58 Kroll P. Psychoses associated with marijuana use in Thailand. J Nerv Ment Dis 1975;161(3):149-56

59 Knight F. Role of cannabis in psychiatric disturbance. Ann N Y Acad Sci 1976; 282:64-71

60 Johnson BA. Psychopharmacological effects of cannabis. Br J Hosp Med 1990; 43(2):114-6

61 Thomas $\mathrm{H}$. Psychiatric symptoms in cannabis users. Br $\mathbf{J}$ Psychiatry 1993;163:141-9

62 Solomons K, Neppe VM, Kuyl JM. Toxic cannabis psychosis is a valid entity. S Afr Med J 1990; 78(8):476-81

63 Imade AG, Ebie JC. A retrospective study of symptom patterns of cannabis-induced psychosis. Acta Psychiatr Scand 1991; 83(2):134-6

64 Kristensen FW. Cannabis and psychoses. Ugeskr Laeger 1994; 156(19):2875-8

65 McGuire PK, Jones P, Harvey I, Bebbington P, Toone B, Lewis S, Murray RM Cannabis and acute psychosis. Schizophr Res 1994; 13(2):161-167

66 Leweke FM, Emrich HM. Carbamazepine as an adjunct in the treatment of schizophrenia-like psychosis related to cannabis abuse. Int Clin Psychopharmacol 1999; 14(1):37-9

67 Van Amsterdam JG, van der Laan JW, Slangen JL. [Cognitive and psychotic effects after cessation of chronic cannabis use]. Ned Tijdschr Geneeskd 1998; 142(10): 504-8 (published erratum appears in Ned Tijdschr Geneeskd 1998; 142(13):752)

68 Caspari D. Cannabis and schizophrenia: results of a follow-up study. Eur Arch Psychiatry Clin Neurosci 999; 249(1):45-9

69 Rubio Valladolid G. Consumo de cannabis en diferentes grupos de población. En Cabrera Forneiro J, Editor de Cannabis ¡Hasta dónde!. Agencia Antidroga, Consejería de Sanidad y Servicios Sociales, Comunidad de Madrid. Ediciones Harcourt, Madrid 1999;12:167-84

70 Varma SL; Sharma I. Psychiatric morbidity in the first-degree relatives of schizophrenic patients. Br J Psychiatry 1993; 162:672-8 [published erratum appears in Br J Psychiatry 1993; 163:700]

71 Hall W. The public health significance of cannabis use in Australia. Aust J Public Health 1995;19(3):235-42

72 McGuire PK; Jones P; Harvey I; Williams M; McGuffin P; Murray RM. Morbid risk of schizophrenia for relatives of patients with cannabis-associated psychosis. Schizophr Res 1995; 15(3)277-81
73 Williams JH, Wellman NA, Rawlins JN. Cannabis use correlates with schizotypy in healthy people. Addiction 1996; 91(6):869-77

74 van Os J, Marcelis M. The ecogenetics of schizophrenia: a review. Schizophr Res 1998; 32(2):127-35

75 Van Os J, Verdoux H. Aspects environnementaux et psychosociaux de la recherche genetique en psychiatrie. Encephale 1998;24(2):125-31

76 Emrich HM, Leweke FM, Scneider V. Towards a cannabinoid hypotesis of schizophrenia: cognitive impairments due to dysregulation of the endogenous cannabinoid system. Pharmacol Biolchem Behav 1997; 56:803-7

77 Schneider U, Leweke FM, Mueller-Vahl KR, Emrich HM. Cannabinoid/anandamide system and schizophrenia: is there evidence for association?. Pharmacopsychiatry 1998;31(Suppl 2):110-3

78 Leweke FM, Giuffrida A, Wurster U, Emrich HM, Piomelli D. Elevated endogenous cannabinoids in schizophrenia. Neuroreport 1999;10(8):1665-9

79 Musty RE, Kaback L. T Relationships between motivation and depression in chronic marijuana users. Life Sci 1995; 56 (23-34): 2151-8

80 Ashizawa T, Saito T, Yamamoto M, Shichinohe S, Ishikawa $\mathrm{H}$, Maeda $\mathrm{H}$ et al. [A case of amotivational syndrome as a residual symptom after methamphetamine abuse]. Nihon Arukoru Yakubutsu Igakkai Zasshi 1996; 31(5): 451-61

81 Okada S, Yamanouchi N, Kodama K, Uchida Y, Hirai S, Sakamoto T, Noda S, Komatsu N, Sato T. Regional cerebral blood flow abnormalities in chronic solvent abusers. Psychiatry Clin Neurosci 1999; 53(3):351-6

82 Kitabayashi Y, Ueda H, Matsuda M, Narumoto J, Kobayashi T, Fukui K. [A case report of organic solvent abuse with amotivational syndrome]. Nihon Arukoru Yakubutsu Igakkai Zasshi 1999; 34(2):130-7

83 Amen DG, Waugh M. High resolution brain SPECT imaging of marijuana smokers with $A D / H D$. J Psychoactive Drugs 1998; 30(2):209-14

84 Struve FA, Patrick G, Straumanis JJ, Fitz-Gerald MJ, Manno J. Possible EEG sequelae of very long duration marihuana use: pilot findings from topographic quantitative EEG analyses of subjects with 15 to 24 years of cumulative daily exposure to THC. Clin Electroencephalogr 1998; 29(1):31-6

85 Struve F.A, Straumanis J.J, Patrick G, Leavitt J, Manno J.E, Manno B.R. Topographic quantitative EEG sequelae of chronic marihuana use: a replication using medically and psychiatrically screened normal subjects. Drug Alcohol Depend 1999;56(3):167-79.

86 McGee R, Williams S, Poulton R, Moffitt T. A longitudinal study of cannabis use and mental health from adolescence to early adulthood. Addiction 2000 Apr 95:491-50.

87 Hall W, Degenhardt L. Cannabis use and psychosis: a review of clinical and epidemiological evidence. Aust N Z J Psychiatry 2000 Feb 34:26-34

88 Hambrecht $M$, Häfner H. Cannabis, vulnerability, and the onset of schizophrenia: an epidemiological perspective. Aust N Z J Psychiatry 2000 Jun 34:468-75 
89 Skosnik PD, Spatz-Glenn L, Park S. Cannabis use is associated with schizotypy and attentional disinhibition. Schizophr Res 2001 Mar 48:83-92

90 Mass R, Bardong C, Kindl K, Dahme B. Relationship between Cannabis Use, Schizotypal Traits, and Cognitive Function in Healthy Subjects. Psychopathology 2001 Jul-Ag; 34(4):209-14
91 Dean B, Sundram S, Bradbury R, Scarr E, Copolov D. Studies on $[3 \mathrm{H}] \mathrm{CP}-55940$ binding in the human central nervous system: regional specific changes in density of cannabinoid-1 receptors associated with schizophrenia and cannabis use. Neuroscience 2001;103(1):9-15

92 Ozaki S, Wada K. [Amotivational syndrome in organic solvent abusers]. Nippon Yakurigaku Zasshi 2001 Jan $117: 42-8$ 